\title{
Допустимые ограничения
}

исключительных прав

на объекты авторского права

В континентальной

\author{
и англо-американской \\ правовых традициях
}

\section{凡目 Е.в. Балабанова}

Преподаватель, кафедра конституционного и административного права факультета права, Национальный исследовательский университет «Высшая школа экономики» (Санкт-Петербург). Адрес: 190008, Российская Федерация, Санкт-Петербург, ул. Союза Печатников, 16. E-mail: evb@spbustavsud.ru

\section{理目 Аннотация}

Статья посвящена наиболее актуальным проблемам функционирования системы допустимых ограничений авторских прав в различных правопорядках. На основе дифференцированного анализа двух правовых традиций в контексте общих тенденций совершенствования правового регулирования могут быть сформулированы следующие выводы: в странах континентального права формирование ограничительного механизма зависит от таких факторов, как характер действия трехступенчатого теста (прямое или опосредованное), порядок применения трехступенчатого теста (кумулятивное или некумулятивное), сочетание основных оценочных критериев (субъектный состав, соответствие определенной цели, доступность произведения, добросовестность использования), наличие альтернативных инструментов. В странах «общего права» формирование ограничительного механизма зависит от наличия общего правила оценки (в рамках доктрины добросовестного использования общее правило заменяют четыре фактора оценки, в рамках доктрины добросовестного использования общее правило оценки допустимости ограничений заменяет критерий добросовестности), дополнительных статутных и нестатутных оценочных критериев (защита публичного интереса, объем и характер использования, обстоятельства и цель использования, возможность достижения цели иным образом и др.). Автор приходит к выводу, что к обстоятельствам, негативно влияющим на эффективность и адаптивность ограничительного механизма, могут быть отнесены такие факторы, как: отсутствие общего правила или возможности его прямого применения; обязательность кумулятивного применения оценочных критериев; отсутствие дополнительных критериев оценки; наличие закрытого перечня ограничений и чрезмерная конкретизация ограничений; ограничение числа целей использования и объектов авторских прав, подлежащих использованию. В статье также доказывается, что проблема чрезмерно жесткой оценки допустимости ограничений может быть решена путем толкования существующих в национальном законодательстве ограничений, а также использования дополнительных квалифицирующих критериев. Формирование гибкого ограничительного механизма упрощает процесс балансировки частных и публичных интересов и актуализации системы огра- 
ничений авторских прав. Особое внимание уделяется исследованию перспектив корректировки правового регулирования, а также проблеме ограничения авторских прав в цифровой среде.

\section{О푤 Ключевые слова}

авторское право; ограничения исключительных авторских прав; защита исключительных прав; ограничительный механизм; трехступенчатый тест; доктрина добросовестного использования; доктрина добросовестного поведения

Для цитирования: Балабанова Е.В. Допустимые ограничения исключительных прав на объекты авторского права в континентальной и англо-американской правовых традициях // Право. Журнал Высшей школы экономики. 2019. № 5. С. 101-120.

УДК: 347.78

DOI: $10.17323 / 2072-8166.2019 .5 .101 .120$

\section{Введение}

В контексте исследования факторов формирования и адаптивных возможностей механизма ограничений исключительных прав на произведения науки, литературы и искусства в рамках континентальной и англо-американской правовых традиций особенный интерес имеет системный анализ инфраструктурных моделей, позволяющих решить проблему отбора критериев, необходимых для оценки допустимости ограничительного воздействия, а также последующая разработка рекомендаций по регулированию уровня гибкости ограничительного механизма с учетом практики изменения его конфигурации. Различие подходов к квалификации ограничений оказывает существенное влияние на вариативность сочетания элементов, составляющих инструментарий, применяемый в процессе оценки нормы на соответствие статусу допустимой. Поэтому принципиальное значение для оценки перспектив балансировки интересов участников складывающихся в рассматриваемой области правоотношений играет возможность актуализации ограничительного механизма с учётом обстоятельств, послуживших причиной его корректировки.

\section{1. Механизм ограничения исключительных прав на результаты интеллектуальной деятельности в рамках континентальной правовой традиции}

Для континентальной правовой традиции характерен ярко выраженный автороцентризм, о чем, в частности, свидетельствует и терминология, используемая для обозначения института авторского права (например, «droit 
d'auteur» или «Urheberrecht»). Такой субъект-ориентированный подход сложился под влиянием идеалистических понятий о фигуре автора, сформировавшихся в эпоху Просвещения и получивших развитие в эпоху романтизма. Очевидно, данное обстоятельство послужило одной из ключевых причин восприятия произведения в качестве продолжения личности автора, свидетельства уникальности человеческого гения. В связи с этим ограничения исключительных прав авторов, первоначально облекавшиеся в форму правил, утверждавших приоритет публичного интереса над частным, вскоре утратили преимущественное значение и приобрели статус некоторой совокупности элементов ограничительного механизма. Это неизбежно привело к пониманию необходимости установления критериев, позволяющих осуществить оценку таких ограничений. Подобные критерии формировались в рамках модели закрытого перечня строго определенных ограничений [Senftleben M., 2013: 1, 2], что во многом способствовало повышению предсказуемости применения ограничительных правил, но не всегда было способно обеспечить возможность широкого доступа к результатам интеллектуальной деятельности.

Анализируя особенности механизма ограничений исключительных авторских прав в континентальных правопорядках, нельзя не учитывать, что принципиальную роль в формировании рассматриваемого механизма сыграл так называемый трехступенчатый тест, имеющий значение некоторого международного стандарта оценки допустимости ограничений.

Правило-прототип, впервые появившееся в Бернской конвенции по охране литературных и художественных произведений ${ }^{1}$ (ст. 9) применительно к ограничению права на воспроизведение, имеет стандартную трехзвенную структуру, включающую условие о допустимости возможного исключения из общего правила при воспроизведении произведений в определенных особых случаях (первый элемент), а также с учетом того, что подобное воспроизведение не наносит ущерба нормальному использованию произведения (второй элемент) и не ущемляет необоснованным образом законные интересы автора (третий элемент).

Та же модель с некоторыми изменениями впоследствии была воспроизведена в Соглашении по торговым аспектам прав интеллектуальной собственности (Соглашение ТРИПС) ${ }^{2}$, в ст. 13 которого для обозначения ограничений используется связка «ограничения и изъятия» (указанные ограничения и изъятия сводятся к некоторым особым случаям, не вступающим в противоречие с обычным использованием произведения и не ущемляющим необоснованно законных интересов правообладателя). Затем указанный инстру-

${ }^{1}$ Бернская Конвенция по охране литературных и художественных произведений от 09.09.1886 // Бюллетень международных договоров. № 9. 2003.

2 Соглашение по торговым аспектам прав интеллектуальной собственности от 15.04.1994 // СЗ РФ. 2012. № 37. С. 2818-2849. 
мент перекочевал в общеевропейское (наднациональное) законодательство, получив развитие в ряде директив EC [Lee N., Westkamp G., Kur A., Ohly A., 2014: 302], включая Директиву о гармонизации определенных аспектов действия авторского права и смежных прав в информационном обществе ${ }^{3}$ (ст. 5). Примечательно, что в некоторых директивах ${ }^{4}$ были воспроизведены исключительно второй и третий элементы теста (предполагалось, вероятно, что первый элемент закрепляется на национальном уровне). При этом не исключена возможность оценки допустимости конкретного ограничения как законодательной, так и судебной властью (соответствующая позиция сформулирована Судом Евросоюза в 2009 г. $\left.{ }^{5}\right)$.

Исследование трансформационных процессов, протекавших в различных европейских странах, свидетельствует о сильном влиянии имплементационных стратегий на итоговый результат применения правила-прототипа. Указанное обстоятельство обусловливается возможностями свободного формирования пула ограничений из установленного на наднациональном уровне перечня, а также адаптации таких ограничений к традициям соответствующих государств. Например, в Греции, Дании, Италии, Франции и ряде других стран общее ограничительное правило имплементировано в национальные законы. С другой стороны, в Австрии, Бельгии, Финляндии и некоторых других странах оно имеет прямое действие и может быть применено судами даже при отсутствии прямых ссылок на него в национальных законах [Westkamp G., 2014: 92-93].

Различается и степень гибкости подходов к оценке допустимости ограничений. Так, в частности, при оценке правила цитирования во Франции применяется строгий подход, допускающий цитирование произведений лишь в случае указания имени автора и источника заимствования при условии, что подобное использование оправдано характером работы (научным, образовательным, информационным и пр.), в которую он включен 6 . В Швейцарии распространен гибкий подход, предполагающий максимально абстрактную формулировку правила цитирования, допускающую возможность трансформации

${ }^{3}$ Directive 2001/29/EC of the European Parliament and of the Council of 22 May 2001 on the harmonisation of certain aspects of copyright and related rights in the information society. OJ L 167, 22.6.2001, pp. 10-19.

${ }^{4}$ Council Directive 91/250/EEC of 14 May 1991 on the legal protection of computer programs. OJ L 111, 5.5.2009, p. 16-22; Council Directive 92/100/EEC of 19 November 1992 on rental right and on certain rights related to copyright in the field of intellectual property. OJ L 346, 27.11.1992, p. 61-66; Directive 2006/115/EC of the European Parliament and of the Council of 12 December 2006 on rental right and lending right and on certain rights related to copyright in the field of intellectual property (codified version). OJ L 376, 27.12.2006, pp. 28-35.

${ }^{5}$ Infopaq International A/S v Danske Dagblades Forening (C-5/08) ECDR 16, 2009. para 56-57.

${ }^{6}$ Intellectual Property Code (1992). Article L122-5(3). Available at: https://www.legifrance.gouv.fr/ content/location/1742 (дата обращения: 14-12-2018) 
исходных текстовых фрагментов без соблюдения требований к указании имени автора, но при условии добросовестного использования произведения в соответствии с поставленной целью7. В Нидерландах применяется смешанный подход, в силу которого цитирование произведений в определенных целях (например, в целях анонсирования и критики) является допустимым при условии публикации произведения с указанием имени автора и источника заимствования, а также соответствия такого использования принятым в обществе обычаям ${ }^{8}$. Иными словами, условия изменения объема отдельных ограничений во многом определяется выбранным законодателем приоритетом в вопросе использования одного из перечисленных способов регулирования.

По уровню гибкости правил оценки определенные различия наблюдаются и в подходах к толкованию трехступенчатого теста. В частности, строгий подход, исключающий расширительное толкование данного инструмента и устанавливающий жесткую привязку существующих в национальном законодательстве ограничений к его содержанию, получил распространение в практике судов Бельгии и Нидерландов, применявших элементы теста на кумулятивной основе. Это зачастую воспринималось как одно из серьезных препятствий на пути выработки гибкой системы ограничений, не ставящей под угрозу свободное использование произведений [Senftleben M., 2004: 181]. Теоретически подобный подход вовсе не исключает возможности изъятия из числа допустимых ограничений традиционных для национальных правопорядков видов использования произведений. Гибкий подход, в противоположность строгому предусматривающий более свободные условия оценки допустимости ограничений, получил широкое распространение в Германии, Швейцарии, Франции и некоторых других европейских странах [Dreier T., 2007: 232]; [Ricketson S., Ginsburg J., 2006: 102].

Таким образом, специфика функционирования механизма ограничений исключительных прав на произведения литературы, науки и искусства во многом предопределяется принятым подходом к оценке допустимости ограничений. При этом очевидно, что фактические границы применения ограничений вполне могут лежать за пределами установленного числа случаев свободного использования результатов интеллектуальной деятельности. В связи с этим на сегодняшний день существуют различные способы, позволяющие решить проблему отсутствия соответствующих ограничений на национальном уровне. В частности, для решения подобной проблемы применяется доктрина предполагаемого согласия, закрепляющая статус допу-

7 Закон Швейцарии об авторском праве и смежных правах (1992), ст. 22. Available at: https:// wipolex.wipo.int/en/legislation/details/648 (дата обращения: 15-12-2018)

${ }^{8}$ Copyright Act (1912). Article 15a. Available at: http://www.wipo.int/wipolex/en/details. jsp?id=12810 (дата обращения: 15-12-2018) 
стимого за ограничением, позволяющим использовать доступное широкой публике произведение в силу предположения о наличии заранее выраженного согласия правообладателя на совершение соответствующих действий 9 . В качестве возможной альтернативы этой доктрине выступает использование трехступенчатого теста при оценке допустимости не только ограничений, но и проверяемых на соответствие таким ограничениям действий пользователей [Kur A., 2008: 33]. К числу иных возможных способов также может быть отнесена доктрина превышения права, признающая недопустимым отклонение от первоначальной цели реализации права [Geiger C., 2013: 171].

Оценивая перспективы применения перечисленных стратегий в контексте корректировки гибкости ограничительного механизма, отметим, что одной из наиболее эффективных с точки зрения адаптивности к требованиям цифровой эпохи может оказаться доктрина предполагаемого согласия, применение которой может быть в значительной степени упрощено в случае законодательного закрепления соответствующей презумпции. Немаловажное значение будет иметь и законодательная универсализация трехступенчатого теста применительно к оценке допустимости конкретных действий пользователей в совокупности с использованием дополнительных оценочных критериев. При этом формализация доктрины превышения права может быть осуществлена путем введения опционального критерия соответствия первоначальной цели использования произведения.

С учетом изложенного представляется, что особенное влияние на формирование механизма допустимых ограничений исключительных прав на произведения могут оказывать следующие факторы: 1) характер действия трехступенчатого теста (прямое действие или включение в ткань национального законодательства); 2) характер его применения (кумулятивное или некумулятивное применение); 3) сочетание ключевых (основных) критериев регулирования уровня его гибкости (субъектный состав, целевое соответствие, доступность широкому кругу лиц и др.), а также возможность применения альтернативных оценочных инструментов.

\section{2. Механизм ограничения исключительных прав на результаты интеллектуальной деятельности в англо-американской правовой традиции}

Англо-американская правовая традиция значительно отличается от континентальной. Здесь авторское право воспринимается как «право на копию»

9 Данная доктрина была применена Верховным Судом ФРГ в одном из дел, связанных с использованием изображений, размещенных в свободном доступе в Интернете, в процессе работы со специальным поисковым сервисом Google. См.: Bundesgerichtshof. I ZR 69/08. 2010. 
или «право на воспроизведение» и носит ярко выраженную ориентацию на коммерциализацию произведений. В странах «общего права» преобладает объект-ориентированный подход, центральное место в котором занимает не автор, а его произведение, что неминуемо накладывает определенный отпечаток на всю систему ограничений исключительных авторских прав. В основе подобного подхода лежит проприетарная кониепиия Дж. Локка [Lock J., 2013: 19], предполагающая восприятие произведения как результата трудовой деятельности автора. В данных странах исключительные права авторов воспринимаются преимущественно в позитивистском ключе; в отношении таких прав распространяется режим срочной монополии, вытекающей из закона. Срочный характер монополии основывается на представлениях о несоответствии вечной монополии правообладателя общесоциальным интересам [Klein B., Moss G., Edwards L., 2015: 12]; [Patterson L.,1968: 202-212].

Рассматриваемая модель формирования ограничительного механизма традиционно основывается на принципе построения системы ограничений открытого типа, в силу которой суды не только оценивают допустимость действий пользователей, но и получают возможность определять границы такой допустимости. В рамках обозначенной модели особое распространение получили две доктрины: доктрина добросовестного использования (американская разновидность) [Senftleben M., 2013: 1, 2] и доктрина добросовестного поведения (британская разновидность) [Bainbridge D., 2009: 5]. Указанные инструменты ориентированы на сохранение таких видов свободного использования, которые являются наиболее значимыми с точки зрения интересов социума [Hua J., 2014: 145, 146]. Как будет показано далее, вторая из перечисленных доктрин, в отличие от первой, практически не подразумевает сохранения возможности отнесения формируемой на ее основе системы ограничений к числу систем открытого типа.

Доктрина добросовестного использования была сформулирована американскими судами в середине XIX века и вскоре обрела популярность, став одним из основных инструментов ограничения исключительных прав [Samuelson P., 2017: 14]. Данная доктрина основывается на критерии разумности использования охраняемых авторским правом результатов интеллектуальной деятельности и предполагает оценку соответствия такого использования факторам допустимости [Patterson L., 1991: 59]. Определение термина, используемого для обозначения доктрины добросовестного использования («fair use») отсутствует в силу вариативности характера и обстоятельств его применения. Это обстоятельство, несомненно, может создавать определенные препятствия для последующего доктринального дискурса с точки зрения разработки некоторого универсального понятия. Вместе с тем сущность доктрины вполне может быть определена при помощи раскрытия содержания 
добросовестного использования через характеристику допустимых (разрешенных) случаев (пределов) свободного (правомерного) использования.

Концептуальные основы данной доктрины были заложены в деле Folsom v. $\mathrm{Marsh}^{10}$, в котором Верховный суд США сформулировал вывод о том, что для признания использования допустимым необходимо произвести проверку такого использования на соответствие следующим параметрам: природе (характеру) и цели использования, количеству и объему используемого материала, наличии препятствий для коммерциализации результата интеллектуальной деятельности, степени сокращения прибыли.

Законодательное закрепление рассматриваемая доктрина получила в ст. 107 Закона об авторском праве $(1976)^{11}$ в виде правила о допустимости свободного использования объектов авторского права при его добросовестном осуществлении. Затем в Законе об авторском праве в цифровую эпоху $(1998)^{12}$ была закреплена четырехзвенная структура оценки, состоящая из следующих факторов: 1) цель и характер использования, 2) характер произведения, 3) количественные и содержательные параметры, 4) уровень потенциального влияния на рынок в контексте дохода от использования произведения. Первый фактор предполагает целевую составляющую, выражающуюся в стимулировании творческой активности, а также общую характеристику использования как коммерческого, так и некоммерческого. Особое внимание при проверке соответствия данному фактору следует уделять не только обоснованию допустимости, но и степени убедительности такого обоснования. Второй фактор демонстрирует зависимость усложнения доказывания добросовестного использования от степени творческого вклада автора в произведение-прототип. Третий фактор связан с исследованием объема и значимости использованного фрагмента произведения с точки зрения совокупного эффекта, производимого на использование произведения-прототипа. Четвертый фактор основывается на оценке дохода, получаемого правообладателем в результате использования произведения.

Помимо перечисленных факторов нельзя не принимать во внимание и факт существования некоторой совокупности нестатутных факторов, сформировавшихся в русле судебной практики. В качестве таких дополнительных оценочных инструментов могут быть указаны, например, добрая совесть или факт неопубликованности произведения, а также фактор защиты общественного интереса [Heins M., Beckles T., 2005: 2].

10 Folsom v. Marsh,. 9 F. Cas. 342 (C.C.D. Mass. 1841).

11 United States Copyright Act (1976). Available at: https://www.copyright.gov/title17/ (дата обращения: 12-12-2018)

12 Digital Millennium Copyright Act (1998). Available at: https://www.copyright.gov/legislation/ dmca.pdf (дата обращения: 15-12-2018) 
В дополнение к статутным и нестатутным критериям для оценки допустимости ограничения исключительных прав могут использоваться и самостоятельные доктрины, в частности, доктрина «грязных рук» (построенная на запрете применения ответных мер вне зависимости от их законности до установления факта нарушения ${ }^{13}$ ), а также доктрина злоупотребления, как видоизмененная разновидность предыдущей доктрины, именуемая иногда доктриной «чистых рук» (построенная на требовании выстраивания линии защиты исключительно в соответствии с принципом справедливости $\left.{ }^{14}\right)$.

Оценивая перспективы гибкого моделирования механизма ограничений авторских прав в контексте доктрины добросовестного использования, особенное внимание следует уделить фактору расширения правовых возможностей по корректировке адаптивности ограничительного инструментария с учетом таких обстоятельств, как активное развитие цифровой среды и стремительный технологический прогресс. В рассматриваемом контексте позитивное влияние доктрины может быть обусловлено преимуществами высокой адаптивности и широкого судебного усмотрения в вопросе толкования ее содержания, а негативное - недостатками размытости формируемого в рамках доктрины правового регулирования и сложности применения составляющих ее основу критериев и факторов. Между тем именно отсутствие некоторой предсказуемости оценки свойства допустимости может служить основанием для более тонкой балансировки интересов участников правоотношений, возникающих в рассматриваемой области.

Доктрина добросовестного поведения (британский вариант) имеет некоторое внешнее сходство с доктриной добросовестного использования - в части допустимости использования результатов интеллектуальной деятельности в разумных целях без согласия правообладателя и выплаты вознаграждения). Она основывается на оценке действий пользователей с точки зрения представлений о том, как поступил бы справедливый и честный человек для достижения соответствующей цели [Colston C., Middleton K., 2005: 312]. Формируемый в русле данной доктрины механизм ограничений исключительных прав на объекты авторского права является более жестким, нежели вариант концепции, существующий в американском праве. Конструкция рассматриваемого механизма демонстрирует отход от традиции использования общих критериев допустимости к применению законодательно закрепленного закрытого перечня ограничительных правил в связке с абстрактным критери-

${ }^{13}$ Motion Picture Patents Co. v. Universal Film Mfg. Co. (243 U.S. 502), 1917; B. Braun Med., Inc. v. Abbott Labs. (124 F.3d 1419, 1426), 1997.

${ }_{14}$ Practice Mgmt. Info. Corp. v. Am. Med. Ass'n (121 F.3d 516, 9th Cir.). 1997; Atari Games Corp. v. Nintendo of Am. Inc. (975 F.2d 832, 846, Fed. Cir.), 1992; Lasercomb Am., Inc. v. Reynolds (911 F.2d 970, 979, 4th Cir.), 1990. 
ем добросовестности, раскрываемым в позициях, формулируемых судебной практикой. Подобное построение структуры используемого для оценки допустимости инструментария, очевидно, обусловливается тем значением, которое имеет в странах общего права проприетарная концепция. В контексте рассматриваемого подхода на смену критериям справедливости и разумности приходит оценка добросовестности. Подобная оценка осуществляется в рамках соответствия конкретного вида использования статутным и нестатутным фактором. К числу последних (нестатутных) могут быть отнесены многочисленные дополнительные критерии (объем, количество цитируемого фрагмента произведения-прототипа, обстоятельства получения и использования произведения, возможность достижения поставленной цели иным образом $\left.{ }^{15}\right)$.

Первоначальное законодательное закрепление рассматриваемой доктрины в Законе об авторском праве $(1911)^{16}$ было ознаменовано простой формулой о допустимости любых форм добросовестного использования любых произведений в определенных целях (частное обучение, исследование, критика, обзор, создание новостных заметок). Впоследствии ограничительный инструментарий корректировался путем вариативного сочетания категорий и условий их применения, а также определенных конкретизирующих критериев, нашедших свое отражение в Законе об авторском праве (1956) и в Законе об авторском праве, промышленных образцах и патентах $(1988)^{17}$. Различные аспекты применения данной доктрины в цифровой среде воплощены в серии сценариев оценки добросовестности использования, заложенной в Руководящих положениях добросовестного поведения в цифровой среде $^{18}$. В условиях постепенной конкретизации ограничительных правил определенное усложнение оценочных конструкций вполне способно оказывать весьма негативное влияние на сохранение необходимого и достаточного уровня гибкости системы оценки допустимости. В связи с этим, видимо, в последнее время наблюдаются попытки распространения характеристик оценочного механизма доктрины добросовестного использования на почву британского варианта концепции [Owen L., 2015: 231]. Между тем, учитывая

${ }^{15}$ Hubbard v. Vosper (1972) 1 All ER 1023, Beloff v. Pressdram (1973) 1 All ER 241, Associated Newspapers Group plc. v. News Group Newspapers Ltd. (1986) RPC 515.

${ }^{16}$ Copyright Act (1911). Available at: http://www.legislation.gov.uk/ukpga/1911/46/pdfs/ukpga_19110046_en.pdf (дата обращения: 14-12-2018)

${ }^{17}$ Copyright Act (1956). Available at: http://www.legislation.gov.uk/ukpga/1956/74/pdfs/ukpga_19560074_en.pdf; Copyright Designs and Patents Act (1988). Available at: http://www.legislation.gov. uk/ukpga/1988/48/pdfs/ukpga_19880048_en.pdf (дата обращения: 14-12-2018)

${ }_{18}$ Guidelines on Fair Dealing in the Electronic Environment. Joint Information Systems Committee and Publishers Association (1998). Available at: https://www.ukoln.ac.uk/services/elib/papers/pa/fair/ (дата обращения: 16-12-2018) 
глобализацию процессов использования результатов творческой деятельности и движения информации, значительным препятствием на пути корректировки системы ограничений может послужить не только отсутствие определенности в понимании и установлении пределов допустимости ограничительного воздействия, но и практическая невозможность конструирования некоторого общего (универсального) механизма оценки свойства допустимости.

Исследование динамических аспектов моделирования ограничительного механизма позволяет прийти к выводу, что постепенное усложнение его структуры вполне способно привести к усилению ограничительного эффекта (за счет конкретизации случаев свободного использования и включения дополнительных условий оценки правомерности действий пользователей).

Сопоставляя обстоятельства возникновения и применения приведенных выше доктрин, можно сформулировать ряд выводов, имеющих значение для определения факторов, влияющих на формирование механизма допустимых ограничений авторских прав в рамках англо-американской правовой традиции. Во-первых, область применения доктрины добросовестного использования несколько шире области применения доктрины добросовестного поведения, поскольку последняя включает в предмет оценки лишь ограниченное число целей и критериев оценки соответствия целям. Во-вторых, доктрина добросовестного использования распространяется на большее число объектов, тогда как доктрина добросовестного поведения применяется в отношении существенно ограниченного формально конкретизированного перечня.

В связи с этим формируемые в результате применения подобных доктрин механизмы ограничений предполагают принципиально различные варианты регулирования (например, для первой из перечисленных доктрин стержневую функцию выполняет общее правило оценки допустимости случаев свободного использования, а для второй - узко сформулированный перечень частных ограничительных правил). Кроме того, отличаются и адаптивные возможности данных доктрин в условиях цифровой среды (при этом, впрочем, не исключена возможность корректировки британского варианта концепции путем законодательного закрепления общего правила оценки добросовестности использования). Таким образом, предполагая, какие обстоятельства могут служить факторами, непосредственно влияющими на формирование ограничительного механизма, особое внимание следует уделить следующим: наличию общего оценочного правила и применимости дополнительных оценочных критериев, включающих статутные и нестатутные факторы.

Вместе с тем нельзя не принимать во внимание и роль, которую играет в процессе балансировки частных и публичных интересов трехступенчатый 
тест [Geiger Ch., Gervais D.J., Senftleen M., 2015: 186, 187]. Очевидно, что его влияние в англо-американском праве с учетом социокультурного контекста и правовых традиций последнего сохраняет слабые позиции. Впрочем, в перспективе это не исключает возможности корректировки системы ограничений. Подобная корректировка может быть произведена при помощи использования трехступенчатого теста в качестве общего оценочного инструмента. Первый, как было показано выше, является субъектоориентированным, подразумевает применение строгого подхода к оценке свойства допустимости ограничений и предполагает использование закрытого перечня ограничений, а второй является объектоориентированным, связан преимущественно с гибким подходом (за исключением британского варианта) и предполагает использование системы ограничений открытого типа и оценку добросовестности действий пользователей.

Отметим, что в последние десятилетия в качестве магистральной тенденции, формирующейся в области оптимизации ограничительного механизма, выступает идея модернизации правового регулирования в направлении повышения адаптивных свойств такого механизма. В связи с этим в науке сформировались устойчивые представления о необходимости следовать идее формирования гибкого ограничительного инструментария с учетом возможных направлений корректировки трехступенчатого теста [Sun H., 2007: 265]. В частности, корректировка теста может быть произведена одним из следующих способов: 1) введением дополнительного критерия балансировки частных и публичных интересов [Suthersanen U., 2005: 121], 2) изменением порядка применения теста (оценка его элементов в качестве дополнительных оценочных факторов) [Geiger Ch., 2006: 697, 698] или 3) созданием расширенного универсализированного международного стандарта оценки (комбинированной структуры, включающей помимо элементов трехступенчатого теста также набор критериев, формируемых в духе доктрины добросовестного использования) [Gervais D., 2005: 1, 29].

Последнее, однако, не исключает необходимости корректировать правовое регулирование, осуществляемое на наднациональном и национальном уровнях. В связи с этим, в частности, весьма интересна концепция сбалансированной интерпретации трехступенчатого теста [Geiger C., Hilty R., Griffiths J., Suthersanen U., 2010: 1-7], основанная на понимании теста в качестве инструмента, способного с максимальной степенью гибкости производить оценку свойства допустимости в контексте целей и задач введения системы ограничений, а также перспектив ее корректировки.

В подобных обстоятельствах более чем естественным является предположение, что использование закрытого перечня ограничений не всегда способно обеспечить стабильность функционирования ограничительного механизма с точки зрения соблюдения баланса частных и публичных инте- 
ресов. Вероятно, в связи с этим уже в начале XXI века в континентальном правовом пространстве зарождаются конструкции, способные расширить традиционные для соответствующей правовой семьи понятия об оценке свойства допустимости. Так, например, в наднациональном европейском законодательстве появляется серия директив ${ }^{19}$, вводящих адаптированные к современному технологическому контексту виды ограничений и исключений, включающие в том числе указания на дополнительные внешние критерии добросовестности, обоснованности и недискриминационности, а также учитывающие важность свободного и широкого доступа к информации.

Немаловажную роль в процессе создания единого подхода к установлению пределов ограничительного воздействия в условиях формирования трансграничной цифровой среды, подразумевающей нивелирование нормативных барьеров в целях перехода к единому правовому инструментарию, с учетом необходимости решения проблем, возникающих ввиду явной эклектичности правового регулирования, занимает поиск адекватных конструктивных моделей оценки допустимости. Ярким примером подобного подхода в континентальном праве выступает так называемая полуоткрытая структура, сформированная на основе ключевых характеристик открытой и закрытой систем ограничений. Подобная конструктивная модель вполне заслуженно оценивается как одна из наиболее перспективных и эффективных с точки зрения ее применимости в континентальных правопорядках [Hugenholtz B., Senftleben M., 2012: 9]. На практике она может быть выражена в виде комбинации общих норм и открытых оценочных критериев (создание в рамках единого стандарта допустимости возможностей для широкого усмотрения суда или же установление приоритета использования достаточного (но не минимального) пула ограничительных правил).

В качестве разновидности подобной структуры может быть рассмотрена модель, предложенная в рамках проекта Европейского кодекса авторского права ${ }^{20}$, соединившего в себе открытость системы ограничений общего права с характерным для континентальной правовой традиции закрытым перечнем ограничений (один из примеров подобного соединения - фиксация правила о применении ограничений по аналогии при соблюдении ряда ус-

19 Directive 2007/65/EC of the European Parliament and of the Council of 11 December 2007 amending Council Directive 89/552/EEC on the coordination of certain provisions laid down by law, regulation or administrative action in Member States concerning the pursuit of television broadcasting activities. OJ L 332, 18.12.2007, P. 27-45; Directive 2010/13/EU of the European Parliament and of the Council of 10 March 2010 on the coordination of certain provisions laid down by law, regulation or administrative action in Member States concerning the provision of audiovisual media services. OJ L 95, 15.4.2010, P. 1-24.

${ }^{20}$ European Copyright Code (текст проекта с разъяснениями). 2010. Available at: https://www. jipitec.eu/issues/jipitec-1-2-2010/2622/wittem-group-european-copyright-code.pdf (дата обращения: 10-12-2018) 
ловий, включая соответствие трехступенчатому тесту). Оценка перспектив применения подобных конструкций со временем приобретает все большее значение ввиду масштабной реформы европейского законодательства, предполагающей реализацию стратегии единого цифрового рынка ${ }^{21}$ и направленной в том числе на корректировку условий свободного использования произведений в связи с активным развитием цифровой среды. В последние годы появилась серия правовых актов ${ }^{22}$, прямо или косвенно затрагивающих вопрос расширения сферы свободного использования, а также демонстрирующих принципиально новый подход к конструированию системы ограничений. Такой подход предусматривает введение определенного набора обязательных ограничений, а также его последовательное расширение (например, вывод из числа нарушений действий, связанных с интеллектуальным анализом данных в процессе функционирования поисковых сервисов).

Исследование материалов оценки регулирующего воздействия, сопровождающие проекты ряда директив ${ }^{23}$ показывает, что идея формирования унифицированного подхода на уровне наднационального законодательства призвана выполнять превентивную функцию в отношении процессов фрагментации национального законодательства. В связи с этим не исключена вероятность того, что кумулятивный эффект реформы в долгосрочной перспективе получит выражение в виде концентрированной совокупности пра-

${ }^{21}$ A Digital Single Market Strategy for Europe. Communication from the Commission to the European Parliament, the Council, the European Economic and Social Committee and the Committee of the Regions. Brussels, 06.05.2015. COM (2015) 192 final.

22 1) Regulation (EU) 2017/1563 of the European Parliament and of the Council of 13 September 2017 on the cross-border exchange between the Union and third countries of accessible format copies of certain works and other subject matter protected by copyright and related rights for the benefit of persons who are blind, visually impaired or otherwise print-disabled. OJ L 242, 20.9.2017, P. 1-5; 2) Regulation of the European Parliament and of the Council laying down rules on the exercise of copyright and related rights applicable to certain online transmissions of broadcasting organisations and retransmissions of television and radio programmes. Brussels, 14.09.2016. COM (2016) 594 final; 3) Directive (EU) 2017/1564 of the European Parliament and of the Council of 13 September 2017 on certain permitted uses of certain works and other subject matter protected by copyright and related rights for the benefit of persons who are blind, visually impaired or otherwise print-disabled and amending Directive 2001/29/ EC on the harmonisation of certain aspects of copyright and related rights in the information society. / OJ L 242, 20.9.2017, P. 6-13; 4) Directive (EU) 2017/1564 of the European Parliament and of the Council of 13 September 2017 on certain permitted uses of certain works and other subject matter protected by copyright and related rights for the benefit of persons who are blind, visually impaired or otherwise printdisabled and amending Directive 2001/29/EC on the harmonization of certain aspects of copyright and related rights in the information society. OJ L 242, 20.9.2017, p. 6-13.

${ }^{23}$ Executive summary of the impact assessment on the modernization of EU copyright rules Accompanying the document Proposal for a Directive of the European Parliament and of the Council on copyright in the Digital Single Market and Proposal for a Regulation of the European Parliament and of the Council laying down rules on the exercise of copyright and related rights applicable to certain online transmissions of broadcasting organizations and retransmissions of television and radio programmes. Commission staff working document. 14.9.2016. SWD (2016) 302 final. 
вил, сочетающих возможности сохранения специфических особенностей национального регулирования и позитивные характеристики универсализированного правового инструментария, направленного на минимизацию рисков неопределенности правового регулирования и непредсказуемости правоприменения.

Краткое исследование особенностей американского и британского вариантов функционирования механизма ограничения исключительных прав на объекты авторского права показывает следующее.

Доктрина добросовестного использования охватывает довольно ограниченную область применения в контексте реализации некоммерческого аспекта свободного использования произведений, лишь фрагментарно затрагивая вопросы нарушения публичных интересов в отрыве от оценки особенностей функционирования рыночных механизмов. В связи с этим нередко выдвигается идея возможности практически полной замены совокупности факторов альтернативными инструментами, в частности, технологическими мерами защиты и договорным регулированием, без ущерба интересам правообладателей и пользователей [Guilbault L., 2002: 85, 86]. Вместе с тем активное использование технологических мер защиты, а также повышение их доступности для правообладателей может существенно осложнить достижение баланса частных и публичных интересов, ограничив таким образом возможности свободного использования до некоторого строго контролируемого сегмента.

Доктрина добросовестного поведения, в отличие от доктрины добросовестного использования, в своей реализации ограничена закрытым перечнем узко сформулированных статутных ограничений, чем приближается по своему характеру к континентальной разновидности ограничительного механизма. Поскольку для правовой традиции стран «общего права» характерен высокий уровень стабильности, какие-либо существенные изменения в конструкции ограничительного механизма в ближайшее время вряд ли возможны. Между тем масштабная цифровизация не может не затронуть и эту доктрину, пока слабо адаптированную под обстоятельства глобального движения информации. В связи с этим в последние годы наблюдаются некоторые попытки законодательного расширения перечня случаев свободного использования, в том числе в направлении обеспечения доступа к цифровому контенту ${ }^{24}$, однако подобные меры носят фрагментарный характер и реализуются преимущественно поступательно, при необходимости.

${ }^{24}$ Modernizing Copyright: A Modern, Robust and Flexible Framework. Government Response to Consultation on Copyright Exceptions and Clarifying Copyright Law. 2011. Available at: http://copyright-debate.co.uk/wp-content/uploads/Modernising-Copyright-a-modern-robust-and-flexible-framework-Government-response.pdf (дата обращения: 09-12-2018); The Copyright (Free Public Showing or Playing) (Amendment) Regulations 2016 (S.I. 2016/565). Available at: https://www.legislation.gov. 


\section{Заключение}

Очевидно, что в рамках континентальной и англо-американской правовых традиций сложились две противоположные тенденции: повышение гибкости ограничительного механизма и его корректировка в сторону последующей конкретизации. При этом на фоне классических тенденций все более активно проявляет себя общая идея баланса частных и публичных интересов с учетом цифровизации и упрощения глобального информационного обмена. Оценивая потенциал применения существующих разновидностей ограничительного механизма, а также их адаптивные возможности, можно прийти к выводу, что к числу ключевых факторов, влияющих на адаптивность подобного механизма, могут быть отнесены следующие: общее правило оценки допустимости или возможность прямого применения такого правила; кумулятивность применения оценочных критериев; дополнительные оценочные критерии; степень открытости системы ограничений и уровень их конкретизации; цели вводимых ограничений, их количество, а также объекты, в отношении которых они распространяются. В связи с этим проблемы корректировки уровня гибкости правового регулирования могут быть решены путем расширительного толкования ограничений на уровне национального законодательства и использования дополнительных квалифицирующих критериев.

\section{Б国 Библиография}

Bainbridge D.I. Intellectual Property. Harlow: Pearson, 2009. 892 p.

Colston C., Middleton K. Modern Intellectual Property Law. London: Cavendish, 2005. $738 \mathrm{p}$.

Dreier T. Regulating Competition by way of Copyright Limitations and Exceptions in Copyright Law. Cheltenham: Edward Elgar, 2007.pp. 232-254.

Geiger C. (2013) The social function of the intellectual property rights, or how ethics can influence the shape and use of IP Law. Dinwoodie B. (ed.). Methods and Perspectives in Intellectual Property. Cheltenham: Edward Elgar, $400 \mathrm{p}$.

uk/uksi/2016/565/pdfs/uksi_20160565_en.pdf (дата обращения: 09-12-2018); The Copyright (Free Public Showing or Playing) (Amendment) Regulations 2016 (S.I. 2016/565). Available at: https://www. legislation.gov.uk/uksi/2016/565/pdfs/uksi_20160565_en.pdf (дата обращения: 09-12-2018); The Enterprise and Regulatory Reform Act 2013 (Commencement No. 10 and Saving Provisions) Order 2016 (S.I. 2016/593). Available at: https://www.legislation.gov.uk/uksi/2016/593/pdfs/uksi_20160593_en.pdf (дата обращения: 09-12-2018); Digital Economy Act (2010). 2016. Available at: https://www.legislation. gov.uk/ukpga/2010/24/pdfs/ukpga_20100024_en.pdf (дата обращения: 09-12-2018); Digital Economy Act (2017). Available at: https://www.legislation.gov.uk/ukpga/2017/30/pdfs/ukpga_20170030_en.pdf (дата обращения: 09-12-2018). 
Geiger C. The Three-Step Test, a Threat to a Balanced Copyright Law? International Review of Intellectual Property and Competition Law, 2006, no 37, pp. 683-699.

Geiger C., Gervais D., Senftleben M. Understanding the three-step test. International Intellectual Property: a Handbook of Contemporary Research. Cheltenham: Edward Elgar, 2015, pp. 167-189.

Geiger C., Hilty R., Griffiths J., Suthersanen U. Declaration of Balanced Interpretation of Three-Step Test in Copyright Law. Available at: https://www.jipitec.eu/issues/ jipitec-1-2-2010/2621/Declaration-Balanced-Interpretation-Of-The-Three-Step-Test. pdf (дата обращения: 17-12-2018)

Gervais D. Towards a New Core International Core International Copyright Norm: the Reverse Three-Step Test. Marquette Intellectual Property Law Review, 2005, vol. 9, pp. 1-35.

Guilbault L. Copyright Limitations and Contracts: An Analysis of the Contractual Overridability of Limitations on Copyright. The Hague: Kluwer Law International, 2002. 377 p.

Heins M., Beckles T. Will Fair Use Survive? Free Expression in the Age of Copyright Control. New York: Brennan Center for Justice, 2005. 67 p.

Hua J. Towards a More Balanced Approach: Rethinking and Readjusting Copyright Systems in the Digital Network Era. Berlin: Springer, 2014. 225 p.

Hugenholtz B., Senftleben M. Fair Use in Europe: in Search of Flexibilities. Amsterdam Law School Research Paper, no 2012-39. Available at: https://www.ivir.nl/publicaties/ download/Fair\%20Use\%20Report\%20PUB.pdf (дата обращения: 10-12-2018)

Klein B., Moss G., Edwards L. Understanding Copyright: Intellectual Property in the Digital Age. London: SAGE, 2015. 160 p.

Kur A. Of Oceans, Islands, and Inland Water - How Much Room for Exceptions and Limitations under the Three Step-Test? Richmond Journal of Global Law and Business, 2008, no 8, pp. 287-350.

Lee N., Westkamp G., Kur A., Ohly A. Intellectual Property, Unfair Competition and Publicity: Convergences and Development. Cheltenham: Edward Elgar, 2014. 368 p.

Lock J. Two Treatises on Government. Manchester: Industrial Systems Research, 2013. $257 \mathrm{p}$.

Owen L. Fair Dealing: a Concept in UK Copyright Law. Learned Publishing, 2015, no 3, pp. 229-231.

Patterson L. Copyright in Historical Perspective. Nashville: Vanderbilt University Press, 1968, pp. 202-212.

Patterson L., Lindberg S. The Nature of Copyright. A Law of User' Rights. Athens: University of Georgia Press, 1991. 274 p.

Ricketson S., Ginsburg J. International Copyright and Neighboring Rights: the Berne Convention and Beyond. Oxford: OUP, 2006. 878 p.

Samuelson P. Justifications for Limitations and Exceptions. Copyright Law in an Age of Limitations and Exceptions. Cambridge: University Press, 2017, pp. 12-59.

Sun H. Overcoming the Achilles Heel of Copyright Law. Northwestern Journal of Technology and Intellectual Property, 2007, vol. 5, pp. 267-331.

Senftleben M. Comparative Approaches to Fair Use: An Important Impulse for Reforms in EU Copyright Law / Dinwoodie G. (ed.). Methods and Perspectives in intellectual property. Cheltenham: Edward Elgar, 2013. 400 p.

Senftleben M. Copyright, Limitations and the Three-step Test. An Analysis of the Threestep Test in International and EC Copyright Law. The Hague: Kluwer Law International, 2004. 340 p. 
Suthersanen U. Human Rights and International Copyright Law. Copyright and Free Speech: Comparative and International Analyses. Oxford: Oxford University Press, 2005. $426 \mathrm{p}$.

Westkamp G. The Implementation of Directive 2001/29/EC in the Member States. London: Centre for Commercial Law Studies, 2007. 482 p.

\section{Pravo. Zhurnal Vysshey Shkoly Ekonomiki. 2019. No 5}

\section{Permissible Restrictions to Copyright in Continental and Anglo-American legal traditions}

\section{凡目 Evgenia Balabanova}

Lecturer, Constitutional and Administrative Law Department, Law Faculty, National Research University Higher School of Economics, Saint Petersburg branch. Address: 16 Souza Pechatnikov Str., Saint Petersburg 190008, Russian Federation. E-mail: evb@ spbustavsud.ru

\section{国国 Abstract}

The article is devoted to the most urgent problems of the functioning of the system of permissible copyright restrictions in different legal orders. Based on the analysis of two legal traditions the following conclusions can be formulated: in civil law countries the formation of a restrictive mechanism depends on such factors as the nature of the threestep test (direct or indirect), the procedure for applying this test (cumulative or noncumulative), the combination of the main evaluation criteria (range of factors, compliance with a specific goal, availability of the work, conscientiousness), availability of alternative tools; in common law countries the formation of such mechanism depends on the existence of a general assessment rule (under the fair use doctrine this rule consists of four factors; under the fair dealing doctrine this rule is replaced by the fairness criterion), additional statutory and non-statutory evaluation criteria (protection of public interest, scope and nature of use, circumstances and purpose of use, the possibility of achieving the goal in another way, etc.). The author comes to the conclusion that such factors as the lack of a general rule or the possibility of its direct application; cumulative application of evaluation criteria; lack of additional criteria; closed list and excessive specification of restrictions; limiting the number of goal and objects have a negative effect on the restrictive mechanism and its adaptability. The article also proves that the problem of excessive rigidity of the assessment system can be solved by the interpretation as well as using additional criteria. The formation of a flexible restrictive mechanism simplifies the process of balancing private and public interests and updating the system of copyright restrictions. A special attention is given to the analysis of some features of legal regulation and to the problem of copyright limitations in the digital environment.

\section{므를 Keywords}

copyright law; permissible restrictions to copyright; protection of exclusive rights; restrictive mechanism; three-step-test; fair use doctrine; fair dealing doctrine. 
For citation: Balabanova E.V. (2019) Permissible Restrictions to Copyright in Continental and Anglo-American Legal Traditions. Pravo. Zhurnal Vysshey shkoly ekonomiki, no 5, pp. 101-120 (in Russian)

DOI: $10.17323 / 2072-8166.2019 .5 \cdot 101.120$

\section{에 References}

Bainbridge D.I. (2009) Intellectual Property. Harlow: Pearson Education, 892 p.

Colston C., Middleton K. (2005) Modern Intellectual Property Law. London: Cavendish, $738 \mathrm{p}$.

Dreier T. (2007) Regulating Competition by Way of Copyright Limitations and Exceptions in Copyright Law. Cheltenham: Edward Elgar, pp. 232-254.

Geiger C. (2013) The social function of the intellectual property rights, or how ethics can influence the shape and use of IP Law. Dinwoodie B. (ed.) Methods and Perspectives in Intellectual Property. Cheltenham: Edward Elgar, $400 \mathrm{p}$.

Geiger C. (2006) Three-Step Test, Threat to Balanced Copyright Law? International Review of Intellectual Property and Competition Law, no 37, pp. 683-699.

Geiger C., Gervais D., Senftleben M. (2015) Understanding the three-step test. Gervais D. (ed.). International Intellectual Property: a Handbook of Contemporary Research. Cheltenham: Edward Elgar, pp. 167-189.

Geiger C., Hilty R., Griffiths J., Suthersanen U. (2010) Declaration of Balanced Interpretation of the Three-Step Test in Copyright Law. Available at: https://www.jipitec.eu/issues/ jipitec-1-2-2010/2621/Declaration-Balanced-Interpretation-Of-The-Three-Step-Test. pdf (accessed: 17-12-2018)

Gervais D. (2005) Towards a New Core International Core International Copyright Norm: the Reverse Three-Step Test. Marquette Intellectual Property Law Review, vol. 9, pp. 1-35.

Guilbault L. (2002) Copyright Limitations and Contracts: An Analysis of the Contractual Overridability of Limitations on Copyright. The Hague: Kluwer Law International, 377 p.

Heins M., Beckles T. (2005) Will Fair Use Survive? Free Expression in the Age of Copyright Control: a policy report. New York: Brennan Center for Justice, $67 \mathrm{p}$.

Hua J.J. (2014) Toward a More Balanced Approach: Rethinking and Readjusting Copyright Systems in the Digital Network Era. Berlin: Springer, $225 \mathrm{p}$.

Hugenholtz B., Senftleben M. (2012) Fair Use in Europe. In Search of Flexibilities. Amsterdam Law School Research Paper, no 2012-39. Available at: https://www.ivir.nl/publicaties/download/Fair\%20Use\%20Report\%20PUB.pdf (accessed: 10-12-2018)

Klein B., Moss G., Edwards L. (2015) Understanding Copyright: Intellectual Property in the Digital Age. London: SAGE, $160 \mathrm{p}$.

Kur A. (2008) Of Oceans, Islands, and Inland Water - How Much Room for Exceptions and Limitations under the Three Step-Test? Richmond Journal of Global Law and Business, no 8, pp. 287-350.

Lee N., Westkamp G., Kur A., Ohly A. (2014) Intellectual Property, Unfair Competition and Publicity: Convergences and Development. Cheltenham: Edward Elgar, 368 p.

Locke J. (2013) Two Treatises of Government. Manchester: Industrial Systems Research, $257 \mathrm{p}$.

Owen L. (2015) Fair Dealing: a Concept in UK Copyright Law. Learned Publishing, no 3, pp. 229-231. 
Patterson L. (1968) Copyright in Historical Perspective. Nashville: Vanderbilt University Press, pp. 202-212.

Patterson L., Lindberg S. (1991) The Nature of Copyright. A Law of User' Rights. Athens: University of Georgia Press, 274 p.

Ricketson S., Ginsburg J. (2006) International Copyright and Neighboring Rights: the Berne Convention and Beyond. Oxford: Oxford University Press, 878 p.

Samuelson P. (2017) Justifications for Limitations and Exceptions. Copyright Law in an Age of Limitations and Exceptions. Cambridge: University Press, pp. 12-59.

Sun H. (2007) Overcoming the Achilles Heel of Copyright Law. Northwestern Journal of Technology \& Intellectual Property, vol. 5, pp. 267-331.

Senftleben M. (2013) Comparative Approaches to Fair Use: An Important Impulse for Reforms in EU Copyright Law. Dinwoodie G. (ed.) Methods and Perspectives in intellectual property. Cheltenham: Edward Elgar, 400 p.

Senftleben M. (2004) Copyright, Limitations and the Three-step Test. An Analysis of the Three-step Test in International and EC Copyright Law. The Hague: Kluwer Law International, $340 \mathrm{p}$.

Suthersanen U. (2005) Human Rights and International Copyright Law. Copyright and Free Speech: Comparative and International Analyses. Oxford: Oxford University Press, $426 \mathrm{p}$.

Westkamp G. (2007) The Implementation of Directive 2001/29/EC in the Member States. London: Centre for Commercial Law Studies, 482 p. 